\title{
Relationship between initial international normalized ratio and prognosis in patients with cardiogenic cerebral embolism
}

\author{
Yigang Liu ${ }^{1}$, Dudu Jiang ${ }^{2}$, Lingjing Jin ${ }^{1}$, Zhiyu Nie ${ }^{1}$ \\ ${ }^{1}$ Department of Neurology, Shanghai Tongji Hospital, Tongji University School of Medicine, Shanghai, China; ${ }^{2}$ Department of Neurology, Seventh \\ People's Hospital Affiliated to Shanghai University of Traditional Chinese Medicine, Shanghai, China \\ Contributions: (I) Conception and design: Y Liu, Z Nie; (II) Administrative support: Z Nie; (III) Provision of study materials or patients: Y Liu, D \\ Jiang, L Jin, Z Nie; (IV) Collection and assembly of data: Y Liu, D Jiang, L Jin, Z Nie; (V) Data analysis and interpretation: Y Liu; (VI) Manuscript \\ writing: All authors; (VII) Final approval of manuscript: All authors. \\ Correspondence to: Zhiyu Nie, MD. Department of Neurology, Shanghai Tongji Hospital, Tongji University School of Medicine, 389 Xincun Road, \\ Shanghai 200065, China. Email: nzhiyu2002@sina.com.
}

Background: Cardiogenic cerebral embolism is one of the most common causes of ischemic stroke. In general, cardioembolic stroke is associated with more severe neurological deficits and higher early mortality, as well as a worse functional outcome. Oral anticoagulant (OAC) therapy could reduce the risk of stroke significantly. However, several limitations have led to it being underused, which raises the failure of anticoagulant therapy. This study aimed to investigate the patients with atrial fibrillation presented cardioembolic stroke who underwent OAC therapy, and to assess treatment efficacy, and outcomes, especially the international normalized ratio (INR) value in the acute phase.

Methods: Clinical data of 306 patients with cardioembolic stroke and etiology of atrial fibrillation were retrospectively analyzed, and demographics, cardiovascular risk factors, embolic cardiopathy, $\mathrm{CHADS}_{2}$ and $\mathrm{CHA}_{2} \mathrm{DS}_{2}$-VASc score, HAS-BLED score, INR value, TOAST subtypes, OCSP classification, modified Rankin Scale (mRS) scores and prognosis were evaluated.

Results: The median score on the CHADS 2 and $\mathrm{CHA}_{2} \mathrm{DS}_{2}$-VASc scales was 3 and 4, respectively; The median score on the HAS-BLED scale was 2 . Only 33 patients $(10.8 \%)$ were in therapeutic INR range at the onset of stroke. In the acute phase, 233 patients $(76.1 \%)$ continued to use OAC therapy, and 73 patients were suspended. Eighteen patients (24.7\%) resumed treatment after an average of 32 days. Thirty-nine of 251 survivors with nonvalvular atrial fibrillation were modified to novel oral anticoagulants (NOACs). At 3 months follow-up, patients with INR $\geq 1.7$ had significantly better prognosis than those with INR $<1.7$, both in the percentage of patients with functional independence $(78.9 \%$ vs. $41.2 \%)$ and in mortality $(7.0 \%$ vs. $25.0 \%)(\mathrm{P}<0.001)$.

Conclusions: Patients presented cardioembolic stroke despite being treated with OAC, especially those with a subtherapeutic INR value, raises the failure of anticoagulant therapy. Despite the ineffectiveness of the OAC, the prognosis is better when the INR $\geq 1.7$ at the initiation of the stroke.

Keywords: Ischemic stroke; cardiogenic cerebral embolism; atrial fibrillation; oral anticoagulants (OACs); international normalized ratio (INR); prognosis

Submitted Oct 13, 2019. Accepted for publication Jul 28, 2020.

doi: 10.21037/apm-19-366

View this article at: http://dx.doi.org/10.21037/apm-19-366 


\section{Introduction}

Cardiogenic cerebral embolism is one of the most common causes of ischemic stroke, accounting for approximately $20 \%$ to $30 \%$ of the total (1). There is a history of nonvalvular atrial fibrillation in about one half the cases, of valvular heart disease in one fourth, and of left ventricular mural thrombus in almost one third (2). In general, cardioembolic stroke is associated with more severe neurological deficits and higher early mortality, as well as a worse functional outcome (3). Therefore, it has led to enormous socio-economic impacts and burdens (4).

Oral anticoagulant (OAC) therapy is currently recommended for the prevention, and multiple clinical trials and meta-analyses have demonstrated that OAC could reduce the risk of stroke by more than $60 \%$, even in patients $>75$ years old (5-7).

Despite strong evidence for the efficacy of the classic OAC warfarin, however, several limitations such as variable dose-response relationship and narrow therapeutic window have led to it being underused $(8,9)$. In order to exert the medication effect, close monitoring with periodical blood tests are required to maintain the international normalized ratio (INR) within the therapeutic range. Meanwhile, lack of adherence to treatment and interactions with drugs and foods often result in patients being under effective INR levels and therefore their risk of stroke rises $(10,11)$. In addition, complications cannot be thoroughly avoided, mainly hemorrhages in different locations of the body, including the brain, which have hindered broader use, especially among the elderly, and have also made the prescription of warfarin challenging for both clinicians and patients (12). According to several studies, the proportion in therapeutic range is approximately $60 \%$ to $70 \%$, which is lower in China $(13,14)$. To understand the risk of hemorrhage and assess the risk/benefit of OAC treatment, some scales such as HAS-BLED (15) scale can be used for assessment, especially for intracranial hemorrhage (16).

The novel oral anticoagulants (NOACs), which do not require periodic blood tests and with a lower interactions rate, could help to optimize the prevention of cardioembolic stroke. In clinical trials, they have shown noninferiority to $\mathrm{OAC}$ in preventing ischemic events and a lower incidence of hemorrhagic complications (17-19). However, its expensive cost and lack of specific antagonists are hampering its prescription (20).

Our objective was to analyze the patients who underwent OAC therapy but still presented cardioembolic stroke, and to assess their etiology, treatment efficacy, and outcomes, especially the INR value in the acute phase. We present the following article in accordance with the STROBE Reporting Checklist (available at http://dx.doi. org/10.21037/apm-19-366).

\section{Methods}

This study was conducted in accordance with the Declaration of Helsinki (as revised in 2013). This study was approved by the Ethics Committee of Shanghai Tongji Hospital (No. SHTJ-2019-044) and individual consent for this retrospective analysis was waived.

We retrospectively reviewed 5,297 patients who were hospitalized in our neurology department with the diagnosis of ischemic stroke during 2014-2017. Of these, a total of 306 patients $(5.8 \%)$ with etiology of atrial fibrillation and underwent OAC treatment (warfarin) at the onset of stroke were analyzed.

The following epidemiological variables of each patient were collected: gender, age, cardiovascular risk factors (hypertension, diabetes, dyslipidemia, and smoking), embolic cardiopathy (including atrial fibrillation, valvulopathy, and cardiomyopathy), $\mathrm{CHADS}_{2}$ score (21), $\mathrm{CHA}_{2} \mathrm{DS}_{2}$-VASc score (22) and HAS-BLED score, INR value in acute phase, stroke etiology [TOAST criteria (23)] and Oxfordshire community stroke project classification (OCSP) classification (24). The condition of the medication use (maintenance or suspension), and reintroduction of the anticoagulant therapy after the suspension, also the use of NOACs were recorded. Similarly, the prognosis was assessed by analyzing baseline status, modified Rankin Scale $(\mathrm{mRS})$ at discharge and 3 months later.

The patient was considered to be within the therapeutic range of anticoagulation if the INR value was 2.0-3.0.

The etiology was determined by vascular examination, including transcranial Doppler, carotid ultrasound, computerized tomography angiogram (CTA) or magnetic resonance angiography (MRA) scan and transthoracic echocardiography (including detection of right-toleft shunts). Telemetry monitoring, transesophageal echocardiography, 24-hour Holter electrocardiography and autoimmune tests were also added when needed.

The neurologists of our department performed the neurological examination and calculated the mRS scale score at the time of discharge and 3 months follow-up.

Statistical analysis was performed using SPSS version 22.0. Chi-square test was used in univariate categorical 
variables and Mann-Whitney $U$ test was used in nonparametric variables. In the regression analysis, the variables related to prognosis were adjusted based on age, cardiovascular risk factors, NIHSS scores, baseline mRS score, and whether received thrombolytic therapy or not. The INR value of 1.7 was defined as a cut-off point by using CUTOFF FINDER software to determine whether the patients had an anticoagulant effect. A value of $\mathrm{P}<0.05$ was considered statistically significant.

\section{Results}

A total of 306 patients' data was collected, with a male: female ratio of $148: 158$. The average hospital stay was $12.58 \pm 8.37$ days (range, $1-78$ days). The mean age at the time of diagnosis was $77.54 \pm 10.93$ years old, and there was no significant difference between genders.

All patients were receiving warfarin treatment for thromboembolism, all of whom had atrial fibrillation (including paroxysmal and permanent), either isolated $(n=291)$ or with other heart diseases $(n=15 ; 4$ cases of cardiomyopathy, 11 cases of valvulopathy). Regarding the presence of cardiovascular risk factors, 56.2\% [172] of patients with hypertension, $24.8 \%$ [76] were diabetics and $20.9 \%$ [64] with dyslipidemia. About 26.5\% [81] had a smoking habit $(72.8 \%$ of them were still active consumption). Similarly, one third [101] had a history of previous ischemic stroke including transient ischemic attack (TIA).

The median score on the $\mathrm{CHADS}_{2}$ and $\mathrm{CHA}_{2} \mathrm{DS}_{2}-\mathrm{VASc}$ scales was 3 and 4 , respectively. In fact, on the $\mathrm{CHA}_{2} \mathrm{DS}_{2}-$ VASc scale, $83.7 \%$ of the patients scored $\geq 2$, which signified high risk of stroke. Regarding the risk of hemorrhage, the median score on the HAS-BLED scale was 2.

The mean INR value in the acute onset phase was $1.89 \pm 0.91 .226$ patients $(73.9 \%)$ presented INR value $<2.0$; 80 patients $(26.1 \%)$ had an INR $\geq 2.0$, ranging from 2.0 to 5.08. Only 33 patients $(10.8 \%)$ were in therapeutic INR range at the onset of stroke.

The median score of the NIHSS scale at baseline was 5 . Regarding the infarct territory, 248 patients (81\%) located in anterior cerebral circulation and $49(16 \%)$ in posterior cerebral circulation, the other $9(3 \%)$ in both circulations.

Thirteen patients underwent endovascular treatment. Twenty patients received intravenous thrombolysis, 10 of them received bridging therapy with intravenous recombinant tissue plasminogen activator prior to endovascular treatment.
Regarding the etiology according to TOAST criteria, all patients attributed to cardioembolic origin. Transesophageal echocardiography was performed in $24.2 \%$ [74] of patients, and thrombosis was found in the left atrial appendage or metal valve in some cases. In no case were significant plaques in the aortic arch or right-left shunts found. In OCSP stroke classification, 137 (44.8\%) patients were classified as partial anterior circulation infarct (PACI), 80 (26.2\%) were considered as total anterior circulation infarct (TACI), $54(17.6 \%)$ were considered as posterior circulation infarct (POCI), and 35 (11.4\%) were lacunar infarct (LACI) (Table 1).

A total of 21 (6.9\%) cerebral hemorrhagic transformations occurred, 8 of which underwent intravenous and/or mechanical thrombolysis. There were 7 cases of parenchymal hematoma $(\mathrm{PH}) 1$ or 2 type.

Regarding OAC treatment, in the acute phase, 233 patients $(76.1 \%)$ continued to use, and the remaining 73 patients were suspended due to hemorrhagic transformation [16], acute stress ulcer [9] or extensive infarction [48].

Of the patients in whom OAC was suspended, 18 (24.7\%) resumed treatment after an average of 32 days (range, 7-85 days). The remaining patients did not restart treatment for the following reasons: a total of 47 patients (64.4\%) died (during hospitalization and follow-up); 8 patients (11.0\%) had a mRS score of 5 or high HAS-BLED score during follow-up (Figure 1).

Of 251 survivors (excluding the dead and un-restarted anticoagulants patients) with nonvalvular atrial fibrillation, only 39 were modified to NOACs (36 dabigatran, 2 rivaroxaban and 1 apixaban).

The degree of dependence was classified based on patient's mRS scale scores: Independent activities of daily living ( $m R S=0-2)$ or functional dependence ( $m R S=3-5)$. At discharge, 163 patients (53.3\%) had mRS 0-2, 105 patients $(34.3 \%)$ were functionally dependent and 38 patients (12.4\%) died during hospitalization.

After discharge, 252 patients were followed-up (38 patients died during hospitalization and 16 cases were lost). At 3 months after stroke, excluding 9 deaths due to stroke recurrence or complications during this period, 70 of the survival patients $(22.9 \%$ of the initial series) remained functionally dependent.

Analyzing the INR at the onset of stroke, the maximum difference could be obtained with the value of 1.7 as the cutoff point. Patients with INR $\geq 1.7$ had significantly better prognosis than those with INR $<1.7$, both in the percentage of patients with functional independence $(78.9 \% \mathrm{vs}$. 
Table 1 Demographic data and baseline characteristics $(\mathrm{n}=306)$

\begin{tabular}{|c|c|}
\hline Characteristics & Value \\
\hline Male: female & $148: 158$ \\
\hline Age mean $\pm S D$ (years) & $77.54 \pm 10.93$ \\
\hline In hospital days & $12.58 \pm 8.37$ \\
\hline \multicolumn{2}{|l|}{ Cardiovascular risk factors } \\
\hline Hypertension (n, \%) & $172(56.2 \%)$ \\
\hline Diabetes (n, \%) & $76(24.8 \%)$ \\
\hline Dyslipidemia (n, \%) & $64(20.9 \%)$ \\
\hline \multirow[t]{2}{*}{ Smoking (n, \%) } & $81(26.5 \%)$ \\
\hline & (56 active) \\
\hline Previous ischemic stroke $(\mathrm{n}, \%)$ & $101(33.0 \%)$ \\
\hline \multicolumn{2}{|l|}{ Cardiopathy } \\
\hline Isolated atrial fibrillation (n, \%) & $291(95.1 \%)$ \\
\hline Cardiomyopathy (n, \%) & $4(1.3 \%)$ \\
\hline Valvulopathy (n, \%) & $11(3.6 \%)$ \\
\hline CHADS2 score on admission (median) & 3 \\
\hline CHA2DS2-VASc score on admission (median) & 4 \\
\hline HAS-BLED score on admission (median) & 2 \\
\hline NIHSS score on admission (median) & 5 \\
\hline INR value on admission & $1.89 \pm 0.91$ \\
\hline INR $<2.0$ & $226(73.9 \%)$ \\
\hline INR $\geq 2.0$ & $80(26.1 \%)$ \\
\hline \multicolumn{2}{|l|}{ OCSP classification } \\
\hline $\mathrm{PACl}$ & $137(44.8 \%)$ \\
\hline $\mathrm{TACl}$ & $80(26.2 \%)$ \\
\hline $\mathrm{POCl}$ & 54 (17.6\%) \\
\hline LACI & 35 (11.4\%) \\
\hline
\end{tabular}

NIHSS, National Institute of Health Stroke Scale; INR, international normalized ratio; OCSP, Oxfordshire community stroke project; $\mathrm{PACl}$, partial anterior circulation infarct; TACI, total anterior circulation infarct; $\mathrm{POCI}$, posterior circulation infarct; LACI, lacunar infarct.

$41.2 \%)$ and in lower mortality $(7.0 \%$ vs. $25.0 \%)(\mathrm{P}<0.001)$ (Figure 2). Even taking the INR value of 2.0 as the cut-off point, which represents that this boundary was considered to be within the effective therapeutic range, there was also significant difference in the prognosis of the stroke when INR $\geq 2.0(\mathrm{P}<0.01)$.

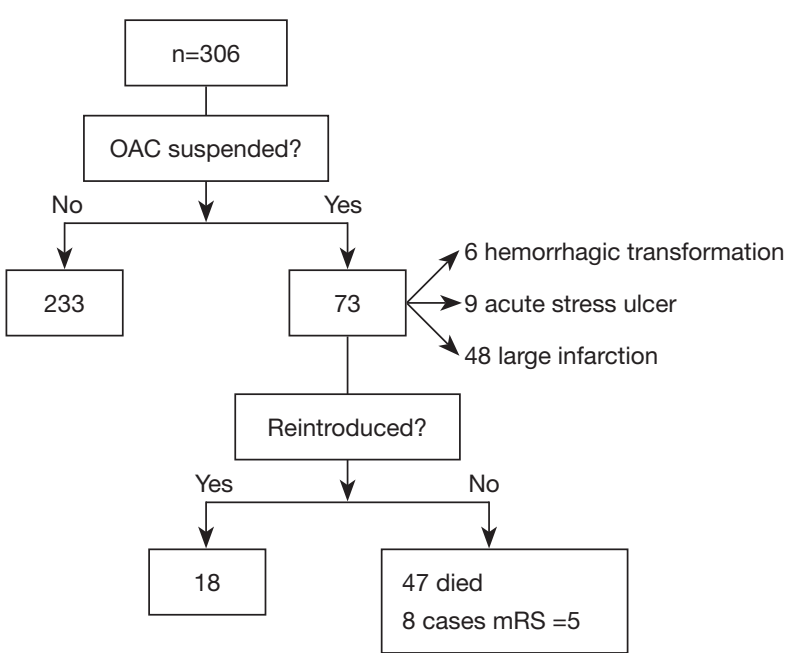

Figure 1 Suspension and reintroduction of oral anticoagulant. OAC, oral anticoagulant; mRS, modified Rankin Scale.

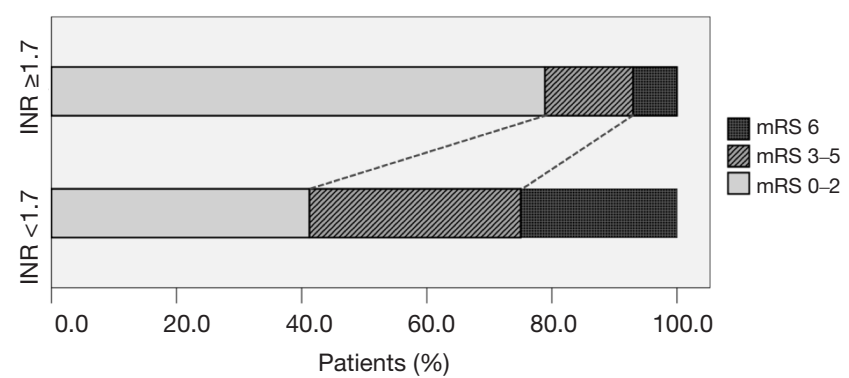

Figure 2 Distribution in percentage relating mRS score at 3 months based on the initial INR value. IMR, international normalized ratio; mRS, modified Rankin Scale.

Regarding the OCSP classification, there was no significant difference in the distribution: TACI (38 vs. 42), PACI (70 vs. 67), POCI (26 vs. 28) and LOCI (17 vs. 18), according to the INR value $(\geq 1.7 v s$. $<1.7)$.

\section{Discussion}

Almost three-quarters of our patients were below the therapeutic INR range at the onset of stroke. Some patients were due to poor medication compliance, the remaining patients, despite proper intake of the drug, still had difficulty maintaining therapeutic levels and, therefore, were inadequately protected from stroke $(10,25)$.

The remaining one-quarter of patients had an INR $\geq 2.0$. Although many patients had one or more cardiovascular risk factors, sometimes it is difficult to determine the 
precise mechanism, the choice of a platelet inhibitor or anticoagulant drug may be difficult, this could not be inferred to be a double pathogenesis of stroke combined with atherothrombosis or an indeterminate cause. Therefore, despite being within the therapeutic range, patients still had a cardioembolic stroke due to underlying heart disease. Patients who had suffered an ischemic stroke with a high-risk source of cardiogenic embolism should generally be treated with anticoagulant drugs to prevent recurrence. In other studies (26), many stroke events were attributed to other causes, which contradicted our conclusion.

In the majority of patients, OAC treatment was maintained on admission because of the high risk of embolism due to their underlying heart disease. Almost all of prescriptions were justified on the basis of their scores of the $\mathrm{CHADS}_{2}$ and $\mathrm{CHA}_{2} \mathrm{DS}_{2}-\mathrm{VASc}$ scales.

Anticoagulant therapy was suspended in patients with hemorrhagic transformation or extensive infarction, but it could be resumed if no hemorrhagic events occurred. There is a paucity of data from large, prospective, randomized studies to answer the appropriate timing of resumption of anticoagulation. It is usually recommended to reintroduce anticoagulation in 1-2 weeks after stroke, except for patients with large infarcts, poor overall neurological function or other risk factors for hemorrhage (27). When maintaining or restarting OAC, the risk of recurrent hemorrhage must be weighed against the risk of an ischemic cerebrovascular event. Both the $\mathrm{CHA}_{2} \mathrm{DS}_{2}$-VASc score and the HASBLED score need to be considered together to determine that the risk of ischemic events is higher than the risk of hemorrhagic events.

The OAC can be reintroduced when without complications, however, the prescriptions of NOACs are still inadequate. According to recent studies, it could be considered that there had been OAC therapeutic failure when patients still presented stroke and NOACs were recommended to use (28). However, only $15.5 \%$ of the candidate survivors had been switched to NOACs in our department. During the last few years, several new NOACs have been developed and approved in clinical applications, they represent important advances over warfarin owing to more predictable pharmacological profiles, fewer drugdrug/food interactions, an absence of major dietary effects, and less risk of intracranial bleeding. However, clinician and patient preference, and especially, the cost may influence the decision to initiate them.

Regarding the evolution, approximately $15.4 \%$ of the patients died between the acute phase and 3 months after stroke, and almost a quarter was functionally dependent at 3 months. These results were consistent with the worse prognosis of cardioembolic stroke (4).

Considering the initial INR, a value $\geq 1.7$ was associated with a better functional prognosis. It had been reported that patients treated with OAC presented smaller size infarcts compared to those who did not, also established an inverse correlation between the INR value at admission and the volume of the lesion on cranial MRI (29). In a recent study, it was observed that INR value $\geq 2$ was also associated with a better prognosis (30). In our study, patients with INR $\geq 2.0$ also had a better prognosis, but the difference was more significant when taking 1.7 as the cut-off point. Curiously, our patients with INR $<1.7$ who received thrombolysis intravenously, did not represent a significant benefit compared to those who did not. It may because these patients had more severe initial neurological deficits (the median NIHSS score was 14) and nearly half of them presented large intracranial vessel occlusion ( $40 \%$ was TACI).

Interventional procedures and complications such as hemorrhagic transformation, which may also influence evolution, were distributed homogeneously in both groups.

The limitations of this study are those inherent in a retrospective analysis, although it has the advantages of a single-center study, which reduce the deviations in the protocol and make the application of treatments and assessments more homogenous. In addition, only taking admitted patients into account, it had not been possible to study some patients with TIA or mild stroke who were treated in the outpatient clinic or transferred to other medical centers. And also, this study had not evaluated the time in therapeutic range (TTR). Thus, we did not assess the history of anticoagulation. These factors may bias our conclusions.

\section{Conclusions}

In conclusion, we highlighted that some patients presented ischemic strokes despite being treated with OAC, especially those with a subtherapeutic INR value, and these stroke events were still cardioembolic etiology, which raises the failure of anticoagulant therapy. Finally, despite the ineffectiveness of the OAC, the prognosis was better when the INR $\geq 1.7$ at the initiation of the stroke.

\section{Acknowledgments}

We thank some members in the Department of Neurology, 
Shanghai Tongji Hospital, Tongji University School of Medicine.

Funding: This study was supported by Priority of Shanghai Key Discipline of Medicine (2017ZZ02020).

\section{Footnote}

Reporting Checklist: The authors have completed the STROBE Reporting Checklist. Available at http://dx.doi. org/10.21037/apm-19-366

Data Sharing Statement: Available at http://dx.doi. org/10.21037/apm-19-366

Conflicts of Interest: All authors have completed the ICMJE uniform disclosure form (available at http://dx.doi. org/10.21037/apm-19-366). The authors have no conflicts of interest to declare.

Ethical Statement: The authors are accountable for all aspects of the work in ensuring that questions related to the accuracy or integrity of any part of the work are appropriately investigated and resolved. This study was conducted in accordance with the Declaration of Helsinki (as revised in 2013). This study was approved by the Ethics Committee of Shanghai Tongji Hospital (No. SHTJ-2019044) and individual consent for this retrospective analysis was waived.

Open Access Statement: This is an Open Access article distributed in accordance with the Creative Commons Attribution-NonCommercial-NoDerivs 4.0 International License (CC BY-NC-ND 4.0), which permits the noncommercial replication and distribution of the article with the strict proviso that no changes or edits are made and the original work is properly cited (including links to both the formal publication through the relevant DOI and the license). See: https://creativecommons.org/licenses/by-nc-nd/4.0/.

\section{References}

1. Petty GW, Brown RD Jr, Whisnant JP, et al. Ischemic stroke subtypes : a population-based study of functional outcome, survival, and recurrence. Stroke 2000;31:1062-8.

2. Cardiogenic brain embolism. The second report of the Cerebral Embolism Task Force. Arch Neurol 1989;46:727-43.

3. Lin HJ, Wolf PA, Kelly-Hayes M, et al. Stroke severity in atrial fibrillation. The Framingham Study. Stroke 1996;27:1760-4.

4. Flint AC, Banki NM, Ren X, et al. Detection of paroxysmal atrial fibrillation by 30-day event monitoring in cryptogenic ischemic stroke: the Stroke and Monitoring for PAF in Real Time (SMART) Registry. Stroke 2012;43:2788-90.

5. Hart RG, Pearce LA, Aguilar MI. Meta-analysis: antithrombotic therapy to prevent stroke in patients who have nonvalvular atrial fibrillation. Ann Intern Med 2007;146:857-67.

6. Mant J, Hobbs FD, Fletcher K, et al. Warfarin versus aspirin for stroke prevention in an elderly community population with atrial fibrillation (the Birmingham Atrial Fibrillation Treatment of the Aged Study, BAFTA): a randomised controlled trial. Lancet 2007;370:493-503.

7. Ruiz Ortiz M, Romo E, Mesa D, et al. Outcomes and safety of antithrombotic treatment in patients aged 80 years or older with nonvalvular atrial fibrillation. Am J Cardiol 2011;107:1489-93.

8. Zimetbaum PJ, Thosani A, Yu HT, et al. Are atrial fibrillation patients receiving warfarin in accordance with stroke risk? Am J Med 2010;123:446-53.

9. Lewis WR, Fonarow GC, LaBresh KA, et al. Differential use of warfarin for secondary stroke prevention in patients with various types of atrial fibrillation. Am J Cardiol 2009;103:227-31.

10. Deitelzweig SB, Buysman E, Pinsky B, et al. Warfarin use and stroke risk among patients with nonvalvular atrial fibrillation in a large managed care population. Clin Ther 2013;35:1201-10

11. Connolly SJ, Pogue J, Eikelboom J, et al. Benefit of oral anticoagulant over antiplatelet therapy in atrial fibrillation depends on the quality of international normalized ratio control achieved by centers and countries as measured by time in therapeutic range. Circulation 2008;118:2029-37.

12. Bungard TJ, Ghali WA, Teo KK, et al. Why do patients with atrial fibrillation not receive warfarin? Arch Intern Med 2000;160:41-6.

13. Baker WL, Cios DA, Sander SD, et al. Meta-analysis to assess the quality of warfarin control in atrial fibrillation patients in the United States. J Manag Care Pharm 2009;15:244-52.

14. Dlott JS, George RA, Huang X, et al. National assessment of warfarin anticoagulation therapy for stroke prevention in atrial fibrillation. Circulation 2014;129:1407-14.

15. Skanes AC, Healey JS, Cairns JA, et al. Focused 2012 update of the Canadian Cardiovascular Society atrial 
fibrillation guidelines: recommendations for stroke prevention and rate/rhythm control. Can J Cardiol 2012;28:125-36.

16. Roldan V, Marin F, Manzano-Fernandez S, et al. The HAS-BLED score has better prediction accuracy for major bleeding than CHADS2 or CHA2DS2-VASc scores in anticoagulated patients with atrial fibrillation. J Am Coll Cardiol 2013;62:2199-204.

17. Connolly SJ, Ezekowitz MD, Yusuf S, et al. Dabigatran versus warfarin in patients with atrial fibrillation. $\mathrm{N}$ Engl J Med 2009;361:1139-51.

18. Patel MR, Mahaffey KW, Garg J, et al. Rivaroxaban versus warfarin in nonvalvular atrial fibrillation. $\mathrm{N}$ Engl J Med 2011;365:883-91.

19. Granger CB, Alexander JH, McMurray JJ, et al. Apixaban versus warfarin in patients with atrial fibrillation. $\mathrm{N}$ Engl J Med 2011;365:981-92.

20. Schulman S. Advantages and limitations of the new anticoagulants. J Intern Med 2014;275:1-11.

21. Gage BF, Waterman AD, Shannon $W$, et al. Validation of clinical classification schemes for predicting stroke: results from the National Registry of Atrial Fibrillation. JAMA 2001;285:2864-70.

22. Camm AJ, Lip GY, De Caterina R, et al. 2012 focused update of the ESC Guidelines for the management of atrial fibrillation: an update of the 2010 ESC Guidelines for the management of atrial fibrillation. Developed with the special contribution of the European Heart Rhythm Association. Eur Heart J 2012;33:2719-47.

23. Ay H, Benner T, Arsava EM, et al. A computerized

Cite this article as: Liu Y, Jiang D, Jin L, Nie Z. Relationship between initial international normalized ratio and prognosis in patients with cardiogenic cerebral embolism. Ann Palliat Med 2020;9(5):2448-2454. doi: 10.21037/apm-19-366 algorithm for etiologic classification of ischemic stroke: the Causative Classification of Stroke System. Stroke 2007;38:2979-84.

24. Bamford J, Sandercock P, Dennis M, et al. Classification and natural history of clinically identifiable subtypes of cerebral infarction. Lancet 1991;337:1521-6.

25. van Walraven C, Jennings A, Oake N, et al. Effect of study setting on anticoagulation control: a systematic review and metaregression. Chest 2006;129:1155-66.

26. Pujol Lereis VA, Ameriso S, Povedano GP, et al. Ischemic stroke in patients with atrial fibrillation receiving oral anticoagulation. J Neurol Sci 2013;334:139-42.

27. Kernan WN, Ovbiagele B, Black HR, et al. Guidelines for the prevention of stroke in patients with stroke and transient ischemic attack: a guideline for healthcare professionals from the American Heart Association/ American Stroke Association. Stroke 2014;45:2160-236.

28. Klijn C, Paciaroni M, Berge E, et al. Antithrombotic treatment for secondary prevention of stroke and other thromboembolic events in patients with stroke or transient ischemic attack and non-valvular atrial fibrillation: A European Stroke Organisation guideline. Eur Stroke J 2019;4:198-223.

29. Ay H, Arsava EM, Gungor L, et al. Admission international normalized ratio and acute infarct volume in ischemic stroke. Ann Neurol 2008;64:499-506.

30. Nakamura A, Ago T, Kamouchi M, et al. Intensity of anticoagulation and clinical outcomes in acute cardioembolic stroke: the Fukuoka Stroke Registry. Stroke 2013;44:3239-42. 